\title{
Proton Hyperpolarization Relay from Nanocrystals to Liquid Water
}

\author{
Naoto Matsumoto, ${ }^{a}$ Koki Nishimura, ${ }^{a}$ Nobuo Kimizuka, ${ }^{a}$ Yusuke Nishiyama,b,c Kenichiro Tateishi, ${ }^{\mathrm{d}}$ \\ Tomohiro Uesaka, ${ }^{\mathrm{d}}$ and Nobuhiro Yanai*,a,e
}

aDepartment of Applied Chemistry, Graduate School of Engineering, Center for Molecular Systems, Kyushu University, 744 Moto-oka, Nishi-ku, Fukuoka 819-0395, Japan.

bNanoCrystallography Unit, RIKEN-JEOL Collaboration Center, Tsurumi, Yokohama, Kanagawa 230-0045, Japan.

cJEOL RESONANCE Inc., 3-1-2 Musashino, Akishima, Tokyo 196-8558, Japan.

dCluster for Pioneering Research, RIKEN, RIKEN Nishina Center for Accelerator-Based Science, Wako, Saitama 3510198, Japan.

ePRESTO, JST, Honcho 4-1-8, Kawaguchi, Saitama 332-0012, Japan.

\begin{abstract}
Dynamic nuclear polarization (DNP) using transient electron spin polarization generated by photoexcitation can improve nuclear magnetic resonance (NMR) sensitivity far beyond the thermal equilibrium limit for analysis in life science and drug discovery. However, DNP of liquid water at room temperature remains an important challenge. In previous studies, polarization has been transferred directly from the electron spins in the solid to the nuclear spins of the target, and this has been limited to near-surface solid or highly-viscous targets. Here, we propose a new method called hyperpolarization relay, in which the polarization of electron spins is transferred to proton spins in the nanocrystals and then to proton spins in bulk water by the nuclear Overhauser effect (NOE). Molecular nanocrystals doped with a polarizing agent that generates a highlypolarized photoexcited triplet were synthesized by a reprecipitation method while controlling the size of the nanocrystals. As the size of the nanocrystals decreases, the efficiency of polarization transfer from nanocrystals to water was improved due to the increase in the surface area.
\end{abstract}

While nuclear magnetic resonance (NMR) spectroscopy is indispensable in a wide range of fields from chemistry to medicine, NMR suffers from inherently poor sensitivity because of low nuclear spin polarization. Dynamic nuclear polarization (DNP), in which the electron spin polarization is transferred to the nuclear spins, is one of the most promising methods to improve the sensitivity of NMR. ${ }^{1-8}$ Water is an extremely attractive and ubiquitous target because the hyperpolarization of water protons can lead to highly sensitive NMR of proteins and biomolecules. ${ }^{9-15}$ Although DNP of water has been reported using electron spin in thermal equilibrium as a polarization source at cryogenic or room temperature, the presence of paramagnetic species shortens the spin-lattice relaxation time $\left(T_{1}\right)$ of water protons and affects the resolution of NMR. ${ }^{16-17}$ Furthermore, the obtainable nuclear spin polarization is limited to the electron spin polarization in the thermal equilibrium, and room-temperature DNP of water is inevitably inferior to that by DNP at cryogenic temperatures.

The use of non-equilibrium electron spin polarization generated by photoexcitation has the great potential to enable significantly high nuclear spin polarization even at room temperature. The pioneering studies have been reported on target nuclear spin hyperpolarization using nonequilibrium electron spin polarization in the nitrogen-vacancy (NV) center of diamonds. ${ }^{18-23}$ However, since the polarization is directly transferred from the electron spins of the NV center to the target nuclear spins by the solid effect, the polarizable region is limited to a small scale near the diamond surface and the polarizable target is limited to solids or highly-viscous liquids. Therefore, how to transfer the polarization from non-equilibrium electron spins in solids to the nuclear spins in bulk liquid water is an extremely important but unsolved problem.

Here, we report the nuclear spin hyperpolarization of bulk liquid water by a new strategy named hyperpolarization relay, in which the photo-generated transient electron spin polarization is transferred to the nuclear spins within nanocrystals, and then transferred to the nuclear spins of liquid water through nuclear Overhauser effect (NOE) (Figure 1). As the nanocrystal size decreases, the polarization transfer by NOE to water occurs more efficiently.

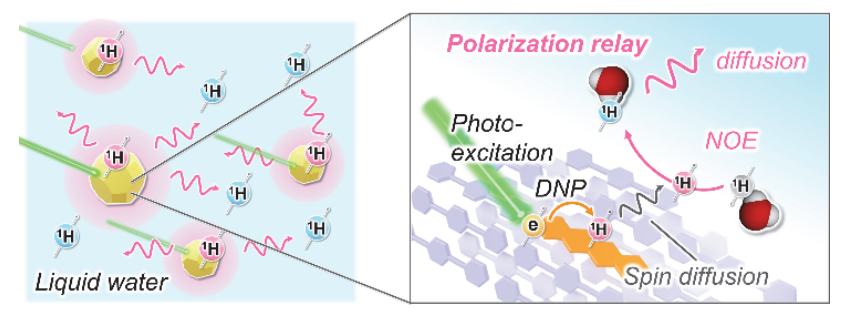

Figure 1. Schematic representation of hyperpolarization relay from nanocrystals to bulk liquid water. DNP: dynamic nuclear polarization, NOE: nuclear Overhauser effect. 
As a proof of concept, we employed organic nanocrystals that are rich in protons and can be hyperpolarized at room temperature by DNP based on photo-excited triplet state (triplet-DNP, Figure 2a). ${ }^{24-27}$ In triplet-DNP, spin-polarized triplet electrons are generated by spin-selective intersystem crossing (ISC) after photoexcitation. This non-equilibrium electron spin polarization is transferred to nuclear spins by integrated solid effect (ISE) under microwave irradiation and magnetic field sweep. ${ }^{24}$ While the application of triplet-DNP had been limited to the solid-state and dissolved solids, ${ }^{28-34}$ our group has previously reported the triplet-DNP even in liquid water. ${ }^{35}$ In our previous work, we prepared pentacene-doped $p$-terphenyl nanocrystals by ball-milling in the presence of a surfactant, cetyltrimethylammonium bromide (CTAB). Proton hyperpolarization by triplet-DNP was observed for the nanocrystals but not for water, probably due to the spin relaxation in CTAB on the nanocrystal surface. Due to the poor solubility of pentacene in common solvents, the only way to prepare the nanocrystals was the ball milling method with surfactants.

(a)

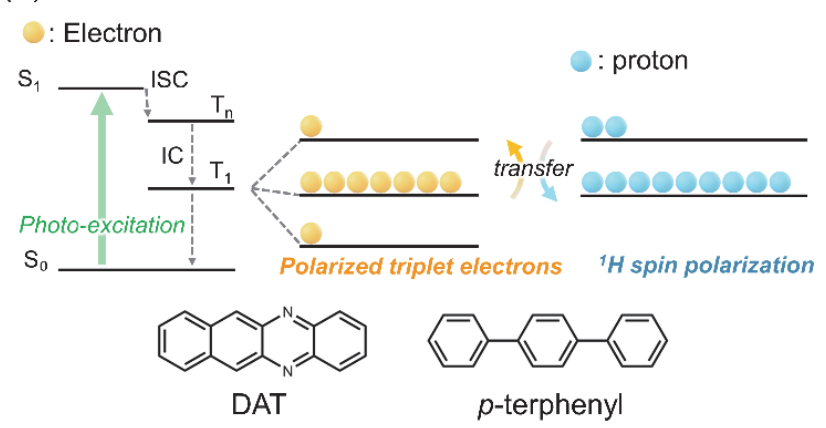

(b)

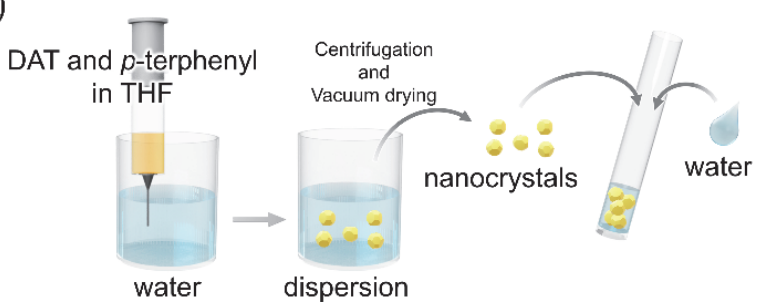

Figure 2. (a) Typical scheme of triplet-DNP and chemical structures of DAT (polarizing agent) and $p$-terphenyl (matrix). ISC: intersystem crossing, IC: Internal conversion. (b) Schematic representation of the preparation of DAT-doped $p$-terphenyl nanocrystals by the reprecipitation method and the sample preparation for triplet-DNP.

We solved this problem by using 5,12-diazatetracene (DAT) as a polarizing agent, which has been previously developed by our group and shows excellent solubility in various organic solvents. ${ }^{36}$ We were able to prepare DATdoped $p$-terphenyl nanocrystals without using surfactants by the reprecipitation method (Figure 2b). ${ }^{37-39} \mathrm{~A}$ THF solution of $p$-terphenyl $(10 \mathrm{mM})$ and DAT was quickly injected into vigorously stirred water in the ice bath. The volume ratio of THF:water was changed as 1:5, 1:10, and 1:100 to control the nanocrystal size. To control the doping amount of DAT, the DAT concentration was tuned to $0.5 \mathrm{mM}$ for THF:water ratio of $1: 5$ and $1: 10$, and $0.25 \mathrm{mM}$ for $1: 100$. After $30 \mathrm{~s}$ of stirring, the formed nanocrystals were isolated by centrifugation. The size of nanocrystals was characterized by scanning electron microscopy (SEM) and dynamic lite scattering (DLS) measurements. SEM observation showed rounded nanoparticles, which decreased in size as the volume ratio of water to THF was increased (Figure 3a, S3). A similar trend was observed in DLS with average sizes of 390, 270, and $170 \mathrm{~nm}$ with the increase of the volume ratio of water to THF (Figure 3b). Nanocrystals with these particle sizes are referred to as $\mathrm{NC}_{390}, \mathrm{NC}_{270}$, and $\mathrm{NC}_{170}$, respectively. Powder X-ray diffraction (PXRD) patterns showed that the crystallinity was well maintained in the nanocrystals compared to bulk $p$-terphenyl crystals doped with $0.05 \mathrm{~mol} \%$ of DAT prepared by melt quenching method (Figure S4). ${ }^{36}$

(a)

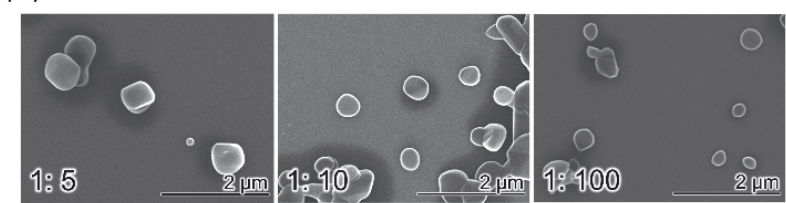

(b)

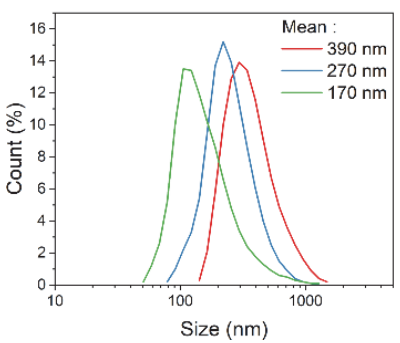

(c)

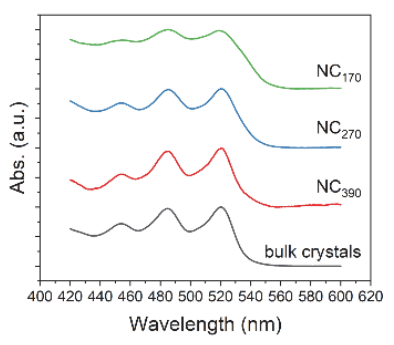

Figure 3. (a) SEM images of nanocrystals obtained by the reprecipitation method with the THF:water volume ratio of 1:5 (left), 1:10 (middle), and 1:100 (right). (b) DLS profiles of the aqueous dispersions of DAT-doped $p$-terphenyl nanocrystals prepared with the THF:water volume ratio of 1:5 (red line), 1:10 (blue line), and 1:100 (green line). (c) UV-vis absorption spectra of DAT-doped $p$-terphenyl bulk crystals (black line) and nanocrystals prepared with the THF:water volume ratio of 1:5 ( $\mathrm{NC}_{390}$, red line), 1:10 $\left(\mathrm{NC}_{270}\right.$, blue line), and 1:100 $\left(\mathrm{NC}_{170}\right.$, green line).

Triplet-DNP requires the molecularly dispersed polarizing agent to avoid the relaxation of electron spin polarization. ${ }^{27}$ DAT in bulk $p$-terphenyl crystals showed the $\pi-\pi^{*}$ absorption peaks at 455, 485, and $520 \mathrm{~nm}$ (Fig. 3c). ${ }^{36}$ The absorption peak positions were almost maintained for DAT in the nanocrystals, suggesting the good dispersibility of DAT in the nanocrystals. The amounts of doped DAT in $\mathrm{NC}_{390}$, $\mathrm{NC}_{270}$, and $\mathrm{NC}_{170}$ were calculated to be $0.038,0.096$, and $0.090 \mathrm{~mol} \%$, respectively, by UV-vis absorption measurements after dissolving the nanocrystals in THF (Figure S2).

The electron spin polarization in photo-excited triplet state was investigated by the X-band time-resolved electron spin resonance (TR-ESR) measurements of the dried nanocrystals at room temperature under pulsed photo-excitation at $527 \mathrm{~nm}$. The spectra of each nanocrystal showed the typical shape of the DAT triplet (Figure S5). ${ }^{36}$ Simulations using the EasySpin toolbox in MATLAB ${ }^{40}$ yielded similar zero-field splitting parameters and relative populations for DAT in bulk crystals and nanocrystals (Table S1). The ESR 
decay time constant was also maintained in the nanocrystals at around $10 \mu$ s (Figure S5, Table S1), which is long enough for polarization transfer to nuclear spins through ISE. 36,41

The ${ }^{1} \mathrm{H}-\mathrm{NMR}$ signal intensity was enhanced by tripletDNP based on the ISE sequence (Figure 4a). After photoexcitation with a pulsed $527 \mathrm{~nm}$ laser at a repetition rate of $800 \mathrm{~Hz}$, microwave was irradiated $(17.6 \mathrm{GHz}, 50 \mathrm{~W})$ while sweeping magnetic field (30 $\mu \mathrm{s})$ to transfer polarization from triplet electron spins to nuclear spins. After repeating this process to accumulate the proton spin polarization, the sample was moved from a resonator to a solenoid coil within $1 \mathrm{~s}$, and the ${ }^{1} \mathrm{H}-\mathrm{NMR}$ signal was detected by using a magic echo sequence at $0.66 \mathrm{~T}(28.2 \mathrm{MHz})$. During the triplet-DNP process, cooled air of ca. $15^{\circ} \mathrm{C}$ was flowed into the resonator to prevent water evaporation due to microwave and laser irradiation. The nanocrystals were mixed with water of $10 \% \mathrm{H}_{2} \mathrm{O}$ in $\mathrm{D}_{2} \mathrm{O}$ in the weight ratio of $\mathrm{NC}$ : water $=$ $1: 4$.

(a)

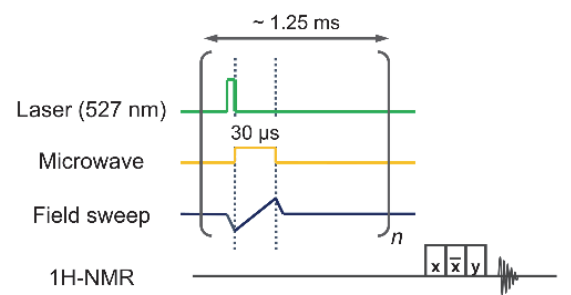

(b)

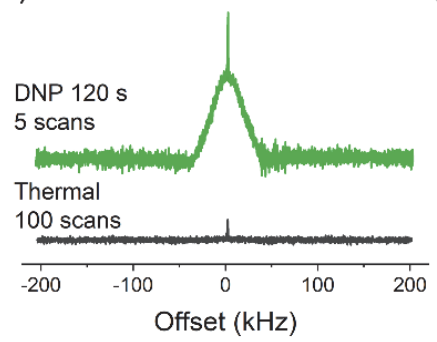

(c)

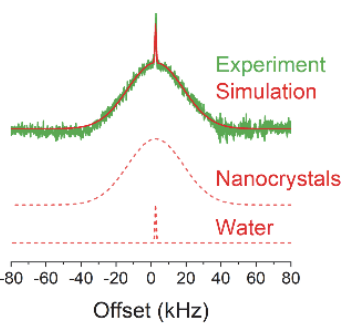

Figure 4. (a) Pulse sequence of triplet-DNP process. (b) ${ }^{1} \mathrm{H}$ NMR spectra of the mixture of $\mathrm{NC}_{170}$ and water at thermal equilibrium (black line) and after triplet-DNP for $120 \mathrm{~s}$ (green line). (c) Enlarged ${ }^{1} \mathrm{H}-\mathrm{NMR}$ spectra (green line) and their fitting results with Gaussian functions (red lines). ${ }^{35}$

In the ${ }^{1} \mathrm{H}$ NMR spectrum for the mixture of $\mathrm{NC}_{170}$ and water at thermal equilibrium, only a sharp peak from water was observed. Notably, after the triplet-DNP sequence for $120 \mathrm{~s}$, the intensity of the water-derived sharp peak clearly increased in addition to the enhanced nanocrystal-derived broad peak. (Figure 4b). By fitting the NMR spectra with the Gaussian function, ${ }^{35}$ the enhancement factor $(\varepsilon)$ of nanocrystals and water was estimated as $103.6 \pm 10.0$ and $2.4 \pm$ 0.3 times, respectively, as the average of three independent measurements (Figure 5a, b, Table 1 ). We finally succeeded in hyperpolarization of bulk liquid water, which has not been achieved by DNP using photo-induced non-equilibrium electron spins in solids.

The single-exponential fitting of the buildup curve for the broad nanocrystal-derived component gave a time constant of $T_{\mathrm{B}, \mathrm{NC}}=24.5 \pm 1.1 \mathrm{~s}$ (Figure $5 \mathrm{a}$ ), which is mostly dominated by the signal decay time constant of the nanocrystals $\mathrm{NC}_{170}$
$\left(T_{\mathrm{D}, \mathrm{NC}}=30.9 \pm 0.4 \mathrm{~s}\right.$, Figure S6). Importantly, the time constants of buildup $\left(T_{\mathrm{B}}\right)$ and decay $\left(T_{\mathrm{D}}\right)$ for the sharp waterderived component were $T_{\mathrm{B} \text {, water }}=19.8 \pm 4.2 \mathrm{~s}$ and $T_{\mathrm{D} \text {, water }}=$ $17.1 \pm 1.5 \mathrm{~s}$ (Figure $5 \mathrm{~b}, \mathrm{S6}$ ), being apparently longer than $T_{1}$ of water $\left(T_{1}\right.$, water $=7.0 \mathrm{~s}$, Figure S7). These $T_{\mathrm{B} \text {, water }}$ and $T_{\mathrm{D} \text {, water }}$ values are rather close to the time constants of the nanocrystals, supporting the polarization leaked out from the nanocrystals to water.

(a)

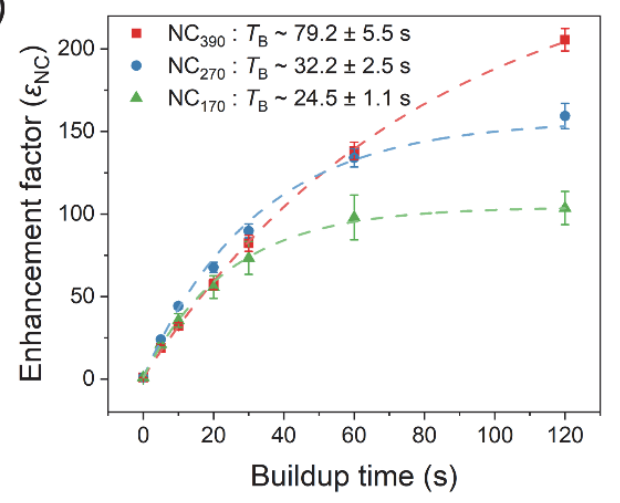

(b)

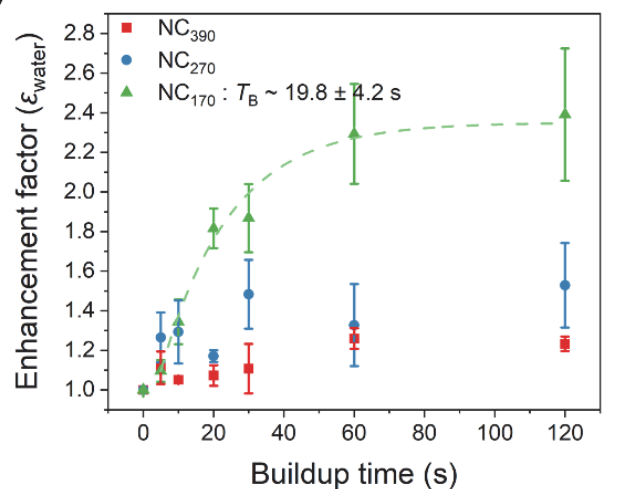

(c)

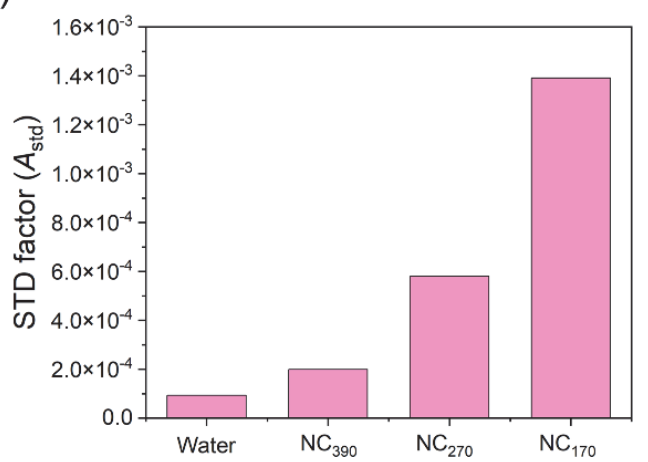

Figure 5. Polarization buildup curves with single exponential fitting results of (a) the nanocrystals and (b) water for the mixture of nanocrystals ( $\mathrm{NC}_{390}$ (red), $\mathrm{NC}_{270}$ (blue), and $\mathrm{NC}_{170}$ (green)) and water. The fitting results of water with $\mathrm{NC}_{390}$ and $\mathrm{NC}_{270}$ are not shown due to high uncertainty by low signal enhancement. Points and error bars indicate the average and standard error, respectively, of three independent measurements. (c) The STD factor ( $\left.A_{\text {std }}\right)$ of the mixture of nanocrystals $\left(\mathrm{NC}_{390}, \mathrm{NC}_{270}\right.$, and $\left.\mathrm{NC}_{170}\right)$ and water. Water without nanocrystals was used as a reference. 
Table 1. Enhancement factor $(\varepsilon)$ after triplet-DNP for $120 \mathrm{~s}$ and time constant of buildup $\left(T_{\mathrm{B}}\right)$ and decay $\left(T_{\mathrm{D}}\right)$. $T_{\mathrm{B}, \text { water, }}$ and $T_{\mathrm{D} \text {, water }}$ of water with $\mathrm{NC}_{270}$ and $\mathrm{NC}_{390}$ are not shown due to high uncertainty by low signal enhancement.

\begin{tabular}{|c|c|c|c|c|c|c|}
\hline & $\varepsilon \mathrm{NC}$ & $\varepsilon$ water & $T_{\mathrm{B}, \mathrm{NC}}(\mathrm{s})$ & $T_{\mathrm{B}, \text { water }}(\mathrm{s})$ & $T_{\mathrm{D}, \mathrm{NC}}(\mathrm{s})$ & $T_{\mathrm{D} \text {,water }}(\mathrm{s})$ \\
\hline $\mathrm{NC}_{390}$ & $205.6 \pm 6.8$ & $1.2 \pm 0.1$ & $79.2 \pm 5.5$ & - & $151.1 \pm 14.5$ & - \\
\hline $\mathrm{NC}_{270}$ & $159.4 \pm 7.7$ & $1.5 \pm 0.2$ & $32.2 \pm 2.5$ & - & $60.3 \pm 5.8$ & - \\
\hline $\mathrm{NC}_{170}$ & $103.6 \pm 10.0$ & $2.4 \pm 0.3$ & $24.5 \pm 1.1$ & $19.8 \pm 4.2$ & $30.9 \pm 0.4$ & $17.1 \pm 1.5$ \\
\hline
\end{tabular}

The transfer of proton spin polarization from the nanocrystal surface to water was also suggested by the size dependence of the nanocrystals. As the nanocrystal size increased from $170 \mathrm{~nm}\left(\mathrm{NC}_{170}\right)$ to $270 \mathrm{~nm}\left(\mathrm{NC}_{270}\right)$ and $390 \mathrm{~nm}$ $\left(\mathrm{NC}_{390}\right)$, the enhancement of water polarization $\left(\varepsilon_{\text {water }}\right)$ decreased from $2.4 \pm 0.3$ to $1.5 \pm 0.2$ and $1.2 \pm 0.1$ times (Figure $5 \mathrm{~b}$, Table 1$)$. This trend supports that the polarization transfer occurs at the interface between the nanocrystals and water. On the other hand, the polarization enhancement of the nanocrystals $\left(\varepsilon_{\mathrm{NC}}\right)$ was lower with decreasing nanocrystal size, mainly due to the shorter spin-lattice relaxation time of the nanocrystals corresponding to $T_{\mathrm{D}, \mathrm{NC}}$ (Table 1, Figure S6). The PXRD patterns of the nanocrystals did not show significant differences between the different nanocrystal sizes (Figure S4). It is assumed that there are some structural disorders near the nanocrystal surface. The future challenge is to fabricate nanocrystals with longer $T_{1}$ while reducing the crystal size.

The size-dependent magnetization transfer from the nanocrystals to water was also supported by saturation transfer difference (STD) NMR measurements at $16.5 \mathrm{~T}$ $(700 \mathrm{MHz}$ ). The magnetization transfer was examined by the extent to which the water signal was reduced by selective saturation of nanocrystal protons. ${ }^{42-45}$ The STD factor $\left(A_{\text {std }}\right)$ was calculated as follows, ${ }^{43,45}$

$$
A_{\text {std }}=\frac{I_{0}-I_{\text {sat }}}{I_{0}}=I_{\text {std }} / I_{0}
$$

where $I_{0}$ is the intensity of off-resonance NMR signal, $I_{\text {sat }}$ is the intensity of on-resonance saturated NMR signal, and $\left(I_{0}-I_{\text {sat }}\right)$ is the STD signal. Water was used as a reference. As the nanocrystal size decreased, the STD factor $\left(A_{\text {std }}\right)$ increased (Figure 5c, S8). This result confirms the scenario that magnetization is transferred from nanocrystals to water by NOE and that magnetization transfer is more likely to occur as the size of the nanocrystals decreases.

In conclusion, we demonstrated for the first time the proton polarization of bulk water using non-equilibrium electron spin polarization generated by photoexcitation. We demonstrated the polarization relay that first transfers the polarization from photoexcited triplet electron spins to proton spins within the nanocrystals, and then transfers the polarization to proton spins of water by NOE on the nanocrystal surface. This provides an important strategy for polarization transfer to liquid targets using various solid polarization sources such as diamond NV centers as well as molecular nanocrystals. Further improvement of the $T_{1}$ of nanocrystals and speed-up of the polarization relay from nanocrystals to water would boost the polarization enhancement of water and target molecules in liquid. This would lead to the realization of a continuous hyperpolarized water supply system that will revolutionize life science and drug discovery.

\section{AUTHOR INFORMATION}

\section{Corresponding Author}

*yanai@mail.cstm.kyushu-u.ac.jp

Notes

The authors declare no competing financial interests.

\section{ACKNOWLEDGMENT}

This work was partly supported by the JST-PRESTO program on "Creation of Life Science Basis by Using Quantum Technology" (JPMJPR18GB), JSPS KAKENHI (JP20H02713, JP20K21211, JP20H05676, and JP21J13049), The Shinnihon Foundation of Advanced Medical Treatment Research, the Innovation inspired by Nature Program of Sekisui Chemical Co. Ltd., RIKENKyushu Univ Science and Technology Hub Collaborative Research Program, the RIKEN Cluster for Science, Technology and Innovation Hub (RCSTI), and the RIKEN Pioneering Project "Dynamic Structural Biology".

\section{REFERENCES}

(1) Carver, T. R.; Slichter, C. P., Polarization of Nuclear Spins in Metals. Phys. Rev. 1953, 92, 212-213.

(2) Overhauser, A. W., Polarization of Nuclei in Metals. Phys. Rev. 1953, 92, 411-415.

(3) Hall, D. A.; Maus, D. C.; Gerfen, G. J.; Inati, S. J.; Becerra, L. R.; Dahlquist, F. W.; Griffin, R. G., Polarization-Enhanced NMR Spectroscopy of Biomolecules in Frozen Solution. Science 1997, 276, 930-932.

(4) Ardenkjaer-Larsen, J. H.; Fridlund, B.; Gram, A.; Hansson, G.; Hansson, L.; Lerche, M. H.; Servin, R.; Thaning, M.; Golman, K., Increase in signal-to-noise ratio of $>10,000$ times in liquid-state NMR. Proc. Natl. Acad. Sci. U. S. A. 2003, 100, 10158-10163.

(5) Golman, K.; In't Zandt, R.; Thaning, M., Real-time metabolic imaging. Proc. Natl. Acad. Sci. U. S. A. 2006, 103, 11270-11275.

(6) Day, S. E.; Kettunen, M. I.; Gallagher, F. A.; Hu, D.-E.; Lerche, M.; Wolber, J.; Golman, K.; Ardenkjaer-Larsen, J. H.; Brindle, K. M., Detecting tumor response to treatment using hyperpolarized ${ }^{13} \mathrm{C}$ magnetic resonance imaging and spectroscopy. Nat. Med. 2007, 13, 1382-1387.

(7) Rossini, A. J.; Zagdoun, A.; Lelli, M.; Lesage, A.; Copéret, C.; Emsley, L., Dynamic Nuclear Polarization Surface Enhanced NMR Spectroscopy. Acc. Chem. Res. 2013, 46, 1942-1951.

(8) Jannin, S.; Dumez, J.-N.; Giraudeau, P.; Kurzbach, D., Application and methodology of dissolution dynamic nuclear polarization in physical, chemical and biological contexts. J. Magn. Reson. 2019, 305, 41-50.

(9) Harris, T.; Szekely, O.; Frydman, L., On the Potential of Hyperpolarized Water in Biomolecular NMR Studies. J. Phys. Chem. B 2014, 118, 3281-3290. 
(10) Chappuis, Q.; Milani, J.; Vuichoud, B.; Bornet, A.; Gossert, A. D.; Bodenhausen, G.; Jannin, S., Hyperpolarized Water to Study Protein-Ligand Interactions. J. Phys. Chem. Lett. 2015, 6, 16741678.

(11) Kim, J.; Liu, M.; Hilty, C., Modeling of Polarization Transfer Kinetics in Protein Hydration Using Hyperpolarized Water. J. Phys. Chem. B 2017, 121, 6492-6498.

(12) Kurzbach, D.; Canet, E.; Flamm, A. G.; Jhajharia, A.; Weber, E. M. M.; Konrat, R.; Bodenhausen, G., Investigation of Intrinsically Disordered Proteins through Exchange with Hyperpolarized Water. Angew. Chem. Int. Ed. 2017, 56, 389-392.

(13) Waddington, D. E. J.; Sarracanie, M.; Zhang, H.; Salameh, N.; Glenn, D. R.; Rej, E.; Gaebel, T.; Boele, T.; Walsworth, R. L.; Reilly, D. J.; Rosen, M. S., Nanodiamond-enhanced MRI via in situ hyperpolarization. Nat. Commun. 2017, 8, 15118.

(14) Negroni, M.; Kurzbach, D., Residue-resolved monitoring of protein hyperpolarization at sub-second time resolution. Commun. Chem. 2021, 4, 147.

(15) Dai, D.; Wang, X.; Liu, Y.; Yang, X.-L.; Glaubitz, C.; Denysenkov, V.; He, X.; Prisner, T.; Mao, J., Room-temperature dynamic nuclear polarization enhanced NMR spectroscopy of small biological molecules in water. Nat. Commun. 2021, 12, 6880.

(16) Armstrong, B. D.; Han, S., Overhauser Dynamic Nuclear Polarization To Study Local Water Dynamics. J. Am. Chem. Soc. 2009, 131, 4641-4647.

(17) Ardenkjaer-Larsen, J. H.; Laustsen, C.; Bowen, S.; Rizi, R., Hyperpolarized $\mathrm{H}_{2} \mathrm{O}$ MR angiography. Magn. Reson. Med. 2014, 71, 50-56.

(18) Shagieva, F.; Zaiser, S.; Neumann, P.; Dasari, D. B. R.; Stöhr, R.; Denisenko, A.; Reuter, R.; Meriles, C. A.; Wrachtrup, J., MicrowaveAssisted Cross-Polarization of Nuclear Spin Ensembles from Optically Pumped Nitrogen-Vacancy Centers in Diamond. Nano Lett. 2018, 18, 3731-3737.

(19) Ajoy, A.; Liu, K.; Nazaryan, R.; Lv, X.; Zangara, P. B.; Safvati, B.; Wang, G.; Arnold, D.; Li, G.; Lin, A.; Raghavan, P.; Druga, E.; Dhomkar, S.; Pagliero, D.; Reimer, J. A.; Suter, D.; Meriles, C. A.; Pines, A., Orientation-independent room temperature optical ${ }^{13} \mathrm{C}$ hyperpolarization in powdered diamond. Sci. Adv. 2018, 4, eaar5492

(20) Fernández-Acebal, P.; Rosolio, O.; Scheuer, J.; Müller, C.; Müller, S.; Schmitt, S.; McGuinness, L. P.; Schwarz, I.; Chen, Q.; Retzker, A.; Naydenov, B.; Jelezko, F.; Plenio, M. B., Toward Hyperpolarization of Oil Molecules via Single Nitrogen Vacancy Centers in Diamond. Nano Lett. 2018, 18, 1882-1887.

(21) Broadway, D. A.; Tetienne, J.-P.; Stacey, A.; Wood, J. D. A.; Simpson, D. A.; Hall, L. T.; Hollenberg, L. C. L., Quantum probe hyperpolarisation of molecular nuclear spins. Nat. Commun. 2018, 9,1246

(22) Healey, A. J.; Hall, L. T.; White, G. A. L.; Teraji, T.; Sani, M.-A.; Separovic, F.; Tetienne, J.-P.; Hollenberg, L. C. L., Polarization Transfer to External Nuclear Spins Using Ensembles of NitrogenVacancy Centers. Phys. Rev. Appl. 2021, 15, 054052.

(23) Tetienne, J.-P.; Hall, L. T.; Healey, A. J.; White, G. A. L.; Sani, M.A.; Separovic, F.; Hollenberg, L. C. L., Prospects for nuclear spin hyperpolarization of molecular samples using nitrogen-vacancy centers in diamond. Phys. Rev. B 2021, 103, 014434.

(24) Henstra, A.; Dirksen, P.; Wenckebach, W. T., Enhanced dynamic nuclear polarization by the integrated solid effect. Phys. Lett. A 1988, 134, 134-136.

(25) Henstra, A.; Lin, T.-S.; Schmidt, J.; Wenckebach, W. T., High dynamic nuclear polarization at room temperature. Chem. Phys. Lett. 1990, 165, 6-10.

(26) Iinuma, M.; Takahashi, Y.; Shaké, I.; Oda, M.; Masaike, A.; Yabuzaki, T.; Shimizu, H. M., Proton polarization with $p$-terphenyl crystal by integrated solid effect on photoexcited triplet state. J. Magn. Reson. 2005, 175, 235-241.

(27) Takeda, K., Triplet State Dynamic Nuclear Polarization: Basics, Concepts, Methods. VDM Publishing: 2009.
(28) Tateishi, K.; Negoro, M.; Nishida, S.; Kagawa, A.; Morita, Y.; Kitagawa, M., Room temperature hyperpolarization of nuclear spins in bulk. Proc. Natl. Acad. Sci. U. S. A. 2014, 111, 7527-7530.

(29) Negoro, M.; Kagawa, A.; Tateishi, K.; Tanaka, Y.; Yuasa, T.; Takahashi, K.; Kitagawa, M., Dissolution Dynamic Nuclear Polarization at Room Temperature Using Photoexcited Triplet Electrons. J. Phys. Chem. A 2018, 122, 4294-4297.

(30) Tateishi, K.; Negoro, M.; Nonaka, H.; Kagawa, A.; Sando, S.; Wada, S.; Kitagawa, M.; Uesaka, T., Dynamic nuclear polarization with photo-excited triplet electrons using 6,13-diphenylpentacene. Phys. Chem. Chem. Phys. 2019, 21, 19737-19741.

(31) Kagawa, A.; Miyanishi, K.; Ichijo, N.; Negoro, M.; Nakamura, Y.; Enozawa, H.; Murata, T.; Morita, Y.; Kitagawa, M., High-field NMR with dissolution triplet-DNP. J. Magn. Reson. 2019, 309, 106623.

(32) Nishimura, K.; Kouno, H.; Kawashima, Y.; Orihashi, K.; Fujiwara, S.; Tateishi, K.; Uesaka, T.; Kimizuka, N.; Yanai, N., Materials chemistry of triplet dynamic nuclear polarization. Chem. Commun. 2020, 56, 7217-7232.

(33) Hamachi, T.; Nishimura, K.; Kouno, H.; Kawashima, Y.; Tateishi, K.; Uesaka, T.; Kimizuka, N.; Yanai, N., Porphyrins as Versatile, Aggregation-Tolerant, and Biocompatible Polarizing Agents for Triplet Dynamic Nuclear Polarization of Biomolecules. J. Phys. Chem. Lett. 2021, 12, 2645-2650.

(34) Eichhorn, T. R.; Parker, A. J.; Josten, F.; Müller, C.; Scheuer, J.; Steiner, J. M.; Gierse, M.; Handwerker, J.; Keim, M.; Lucas, S.; Qureshi, M. U.; Marshall, A.; Salhov, A.; Quan, Y.; Binder, J.; Jahnke, K. D.; Neumann, P.; Knecht, S.; Blanchard, J. W.; Plenio, M. B.; Jelezko, F.; Emsley, L.; Vassiliou, C. C.; Hautle, P.; Schwartz, I., Hyperpolarized Solution-State NMR Spectroscopy with Optically Polarized Crystals. J. Am. Chem. Soc. 2022, 144, 2511-2519.

(35) Nishimura, K.; Kouno, H.; Tateishi, K.; Uesaka, T.; Ideta, K.; Kimizuka, N.; Yanai, N., Triplet dynamic nuclear polarization of nanocrystals dispersed in water at room temperature. Phys. Chem. Chem. Phys. 2019, 21, 16408-16412.

(36) Kouno, H.; Kawashima, Y.; Tateishi, K.; Uesaka, T.; Kimizuka, N.; Yanai, N., Nonpentacene Polarizing Agents with Improved Air Stability for Triplet Dynamic Nuclear Polarization at Room Temperature. J. Phys. Chem. Lett. 2019, 10, 2208-2213.

(37) Kasai, H.; Nalwa, H. S.; Oikawa, H.; Okada, S.; Matsuda, H. Minami, N.; Kakuta, A.; Ono, K.; Mukoh, A.; Nakanishi, H., A Novel Preparation Method of Organic Microcrystals. Jpn. J. Appl. Phys. 1992, 31, L1132-L1134.

(38) Kasai, H.; Kamatani, H.; Okada, S.; Oikawa, H.; Matsuda, H.; Nakanishi, H., Size-Dependent Colors and Luminescences of Organic Microcrystals. Jpn. J. Appl. Phys. 1996, 35, L221-L223.

(39) Chung, H.-R.; Kwon, E.; Oikawa, H.; Kasai, H.; Nakanishi, H., Effect of solvent on organic nanocrystal growth using the reprecipitation method. J. Cryst. Growth 2006, 294, 459-463.

(40) Stoll, S.; Schweiger, A., EasySpin, a comprehensive software package for spectral simulation and analysis in EPR. J. Magn. Reson. 2006, 178, 42-55.

(41) Fujiwara, S.; Matsumoto, N.; Nishimura, K.; Kimizuka, N.; Tateishi, K.; Uesaka, T.; Yanai, N., Triplet Dynamic Nuclear Polarization of Guest Molecules through Induced Fit in a Flexible Metal-Organic Framework. Angew. Chem. Int. Ed. 2022, 61, e202115792.

(42) Mayer, M.; Meyer, B., Characterization of Ligand Binding by Saturation Transfer Difference NMR Spectroscopy. Angew. Chem. Int. Ed. 1999, 38, 1784-1788.

(43) Mayer, M.; Meyer, B., Group Epitope Mapping by Saturation Transfer Difference NMR To Identify Segments of a Ligand in Direct Contact with a Protein Receptor. J. Am. Chem. Soc. 2001, 123, 61086117.

(44) Yan, J.; Kline, A. D.; Mo, H.; Shapiro, M. J.; Zartler, E. R., The effect of relaxation on the epitope mapping by saturation transfer difference NMR. J. Magn. Reson. 2003, 163, 270-276.

(45) Mayer, M.; James, T. L., NMR-Based Characterization of Phenothiazines as a RNA Binding Scaffold. J. Am. Chem. Soc. 2004, $126,4453-4460$. 